July 2000

\title{
Triviality of GHZ operators of higher spin
}

\author{
J. Savinien, J. Taron‡, R. Tarrach \\ Departament d'Estructura $i$ Constituents de la Matèria \\ Facultat de Física, Universitat de Barcelona \\ Diagonal 647, E-08028 Barcelona, Spain \\ and \\ I. F. A. E.
}

\begin{abstract}
We prove that local observables of the set of GHZ operators for particles of spin higher than $1 / 2$ reduce to direct sums of the spin $1 / 2$ operators $\sigma_{x}, \sigma_{y}$ and, therefore, no new contradictions with local realism arise by considering them.
\end{abstract}

*e-mail: taron@ecm.ub.es 


\section{Introduction}

The GHZ theorem [1] provides a powerful test of quantum non-locality, which can be confirmed or refuted by the outcome of just one single experiment [2]. Formulated for three spin $1 / 2$ particles [2] [3], the argument is based on the anti-commutative nature of the $2 \mathrm{x} 2$ spin operators $\sigma_{x}, \sigma_{y}$. The values of the three mutually commuting observables

$$
\sigma_{x}^{a} \otimes \sigma_{y}^{b} \otimes \sigma_{y}^{c} \equiv \sigma_{x}^{a} \sigma_{y}^{b} \sigma_{y}^{c}, \quad \sigma_{y}^{a} \sigma_{x}^{b} \sigma_{y}^{c}, \quad \sigma_{y}^{a} \sigma_{y}^{b} \sigma_{x}^{c},
$$

and their product, $-\sigma_{x}^{a} \sigma_{x}^{b} \sigma_{x}^{c}$, cannot be obtained, consistently, by making local assignments to each of the individual spin operators, $m_{x}^{I}, m_{y}^{I}= \pm 1, I=a, b, c$. This is not a contradiction of Quantum Mechanics: the state $|\psi\rangle=\frac{1}{\sqrt{2}}(|\uparrow \uparrow \uparrow\rangle-|\downarrow \downarrow \downarrow\rangle)$, for instance, is one of the common eigenstates of the four operators, with eigenvalues $\lambda_{1}=\lambda_{2}=\lambda_{3}=1, \lambda_{4}=-1$, respectively. $|\psi\rangle$ is a highly correlated (entangled) state of the three parties which has no defined value for $\sigma_{x}^{I}, \sigma_{y}^{I}$.

In this note we address the question of how to generalize the argument to particles of higher spin and find that there are no non-trivial extensions other than direct sums of operators that can be brought into the form $\sigma_{x}, \sigma_{y}$ by means of local unitarity transformations. (For odd dimensional Hilbert spaces the direct sum is completed by a one-dimensional submatrix, i.e., a c-number in the diagonal). We give a proof for the cases of spin 1 and $3 / 2$. Similar problems have been addressed in [4].

Let us look for observables $A, B$ such that $A B=\omega B A$ (their hermiticity implies that $\omega$ is at most a phase): this is a necessary condition for the commutator relations $\left[A_{1}^{a} A_{2}^{b} A_{3}^{c}, B_{1}^{a} B_{2}^{b} B_{3}^{c}\right]=$ etc... $=0$ to hold. As we shall see, all interesting cases correspond to $\omega=-1$. Without loss of generality, $A$ can always be taken diagonal, $A=\operatorname{diag}\left(\lambda_{1}, \lambda_{2}\right)$, for the simplest case $\mathrm{s}=1 / 2$. The above condition reads

$$
A B-\omega B A=\left(\begin{array}{cc}
(1-\omega) \lambda_{1} b_{11} & \left(\lambda_{1}-\omega \lambda_{2}\right) b_{12} \\
\left(\lambda_{2}-\omega \lambda_{1}\right) b_{12}^{*} & (1-\omega) \lambda_{2} b_{22}
\end{array}\right)=0 .
$$

If $\omega \neq 1$, a solution with non-vanishing off-diagonal elements is allowed if $\omega^{2}=1$, i.e., $\omega=-1$ This leads to

$$
A=\left(\begin{array}{cc}
1 & 0 \\
0 & -1
\end{array}\right), \quad B=\left(\begin{array}{cc}
0 & b \\
b^{*} & 0
\end{array}\right)
$$

which can always be transformed to $\sigma_{x}$ and $\sigma_{y}$, by rotations and adequate normalization. These are the operators of the example (1). For spin 1/2 the set of GHZ operators are in this sense unique.

\section{Spin one}

For higher spins the proof proceeds along the same lines. We find one case of interest, with $\omega=-1$,

$$
A=\left(\begin{array}{ccc}
1 & & \\
& -1 & \\
& & -1
\end{array}\right), \quad B=\left(\begin{array}{ccc}
0 & b & c \\
b^{*} & 0 & 0 \\
c^{*} & 0 & 0
\end{array}\right) \text {. }
$$


In the basis where $B$ is diagonal $A$ and $B$ read

$$
A=-\left(\begin{array}{ccc}
1 & 0 & 0 \\
0 & 0 & 1 \\
0 & 1 & 0
\end{array}\right), \quad B=\sqrt{|b|^{2}+|c|^{2}}\left(\begin{array}{ccc}
0 & & \\
& 1 & \\
& & -1
\end{array}\right)
$$

which proves the assertion in the case of spin one, as a rotation around $x$ brings $B$ into the form $0 \oplus \sigma_{y}$, while $A$ is left as $1 \oplus \sigma_{x}$, up to normalizations.

\section{Spin $3 / 2$}

For spin $3 / 2$, in addition to cases that reduce straightforwardly to those of lower spins, we find:

$$
A=\left(\begin{array}{cccc}
1 & & & \\
& -1 & & \\
& & -1 & \\
& & & -1
\end{array}\right), B=\left(\begin{array}{cccc}
0 & a & b & c \\
a^{*} & 0 & 0 & 0 \\
b^{*} & 0 & 0 & 0 \\
c^{*} & 0 & 0 & 0
\end{array}\right) .
$$

In the basis where $B$ is diagonal $A$ and $B$ read

$$
A=-\left(\begin{array}{cccc}
0 & 1 & 0 & 0 \\
1 & 0 & 0 & 0 \\
0 & 0 & 1 & 0 \\
0 & 0 & 0 & 1
\end{array}\right), \quad B=\sqrt{|a|^{2}+|b|^{2}+|c|^{2}}\left(\begin{array}{cccc}
1 & & & \\
& -1 & & \\
& & 0 & \\
& & & 0
\end{array}\right)
$$

which is again diagonal in two, $2 \times 2$, blocks.

The last case corresponds to

$$
A=\left(\begin{array}{cccc}
1 & & & \\
& -1 & & \\
& & 1 & \\
& & & -1
\end{array}\right), B=\left(\begin{array}{cccc}
0 & a & 0 & b \\
a^{*} & 0 & c^{*} & 0 \\
0 & c & 0 & d \\
b^{*} & 0 & d^{*} & 0
\end{array}\right) .
$$

The following list of unitary transformations bring these matrices to the desired form:

a) With

$$
F=\left(\begin{array}{llll}
1 & 0 & 0 & 0 \\
0 & 0 & 1 & 0 \\
0 & 1 & 0 & 0 \\
0 & 0 & 0 & 1
\end{array}\right)
$$

$F^{\dagger}=F=F^{-1}$, we find

$$
A^{\prime}=F A F=\left(\begin{array}{ll}
I & \\
& -I
\end{array}\right), \quad B^{\prime}=F B F=\left(\begin{array}{ll} 
& \mathcal{B} \\
\mathcal{B}^{\dagger} &
\end{array}\right),
$$

where

$$
\mathcal{B}=\left(\begin{array}{ll}
a & b \\
c & d
\end{array}\right)
$$


b) A unitary transformation of the form $U=\left(\begin{array}{cc}U_{1} & \\ & U_{2}\end{array}\right)$ leaves $A^{\prime}$ invariant and allows to diagonalize $\mathcal{B}$

$$
A^{\prime \prime}=A^{\prime}, \quad B^{\prime \prime}=U B^{\prime} U^{\dagger}=\left(\begin{array}{cc} 
& U_{1} \mathcal{B} U_{2}^{\dagger} \\
\left(U_{1} \mathcal{B} U_{2}^{\dagger}\right)^{\dagger} &
\end{array}\right)=\left(\begin{array}{cccc} 
& m & 0 \\
& & 0 & n \\
& & & \\
m^{*} & 0 & & \\
0 & n^{*} & &
\end{array}\right)
$$

We have used the result that the generic matrix $\mathcal{B}$ can be brought to a diagonal form with two unitary matrices $U_{1}, U_{2}$.

c) Finally, acting with $F$ again,

$$
A^{\prime \prime \prime}=A, \quad B^{\prime \prime \prime}=\left(\begin{array}{cccc}
0 & m & & \\
m^{*} & 0 & & \\
& & 0 & n \\
& & n^{*} & 0
\end{array}\right)
$$

which completes the proof.

\section{Conclusions}

We conclude that the equation $A B=\omega B A$ is very restrictive on $\omega$ and on the possible forms of $\mathrm{A}$ and $\mathrm{B}$; as the Hilbert space dimension increases, with increasing spin, all its solutions for $\omega \neq 1$ have $\omega=-1$ and are essentially direct sums of the two-dimensional $\sigma_{x}$ and $\sigma_{y}$. In this sense there are no solutions that could, in principle, enrich the possibilities opened by the GHZ theorem.

\section{Acknowledgments}

J.S., J.T., R.T. acknowledge the Centro de Ciencias de Benasque for hospitality while this work was beeing done. J.S. also acknowledges the Department ECM for hospitality and financial support. J.T. and R.T. acknowledge financial support by CICYT project AEN 98-0431, CIRIT project 1998 SGR-00026 and CEC project IST-1999-11053. 


\section{References}

[1] D.M. Greenberger, M. Horne, and A. Zeilinger, "Going beyond Bell's theorem" in Bell's Theorem, Quantum Theory, and Conception of the Universe, edited by M.Kafatos (Kluwer Academic, Dordrecht, 1989), pp. 69-72.

[2] N.D. Mermin, Am.J.Phys. 58 (1990) 731.

[3] A. Peres, Quantum Theory: Concepts and Methods, Kluwer Academic Publishers, Dordrecht, 1995).

[4] A. Cabello, unpublished. 